\title{
Acoustic and photonic topological insulators by topology optimization
}

\author{
Christiansen, Rasmus E.; Wang, Fengwen; Stobbe, Søren; Sigmund, Ole
}

Published in:

Proceedings of SPIE - Metamaterials, Metadevices, and Metasystems 2019

Link to article, DOI:

$10.1117 / 12.2528504$

Publication date:

2019

Document Version

Publisher's PDF, also known as Version of record

Link back to DTU Orbit

Citation (APA):

Christiansen, R. E., Wang, F., Stobbe, S., \& Sigmund, O. (2019). Acoustic and photonic topological insulators by topology optimization. In N. Engheta, M. A. Noginov, \& N. I. Zheludev (Eds.), Proceedings of SPIE -

Metamaterials, Metadevices, and Metasystems 2019 (Vol. 11080). [1108003] SPIE - International Society for Optical Engineering. Proceedings of SPIE - The International Society for Optical Engineering https://doi.org/10.1117/12.2528504

\section{General rights}

Copyright and moral rights for the publications made accessible in the public portal are retained by the authors and/or other copyright owners and it is a condition of accessing publications that users recognise and abide by the legal requirements associated with these rights.

- Users may download and print one copy of any publication from the public portal for the purpose of private study or research.

- You may not further distribute the material or use it for any profit-making activity or commercial gain

- You may freely distribute the URL identifying the publication in the public portal 


\section{Acoustic and photonic topological insulators by topology optimization}

Rasmus E. Christiansen, Fengwen Wang, Søren Stobbe, Ole Sigmund

Rasmus E. Christiansen, Fengwen Wang, Søren Stobbe, Ole Sigmund, "Acoustic and photonic topological insulators by topology optimization," Proc. SPIE 11080, Metamaterials, Metadevices, and Metasystems 2019, 1108003 (5 September 2019); doi: 10.1117/12.2528504

SPIE Event: SPIE Nanoscience + Engineering, 2019, San Diego, California, United States 


\title{
Acoustic and photonic topological insulators by topology optimization
}

\author{
Rasmus E. Christiansen ${ }^{1}$, Fengwen Wang ${ }^{1}$, Søren Stobbe ${ }^{2}$, Ole Sigmund ${ }^{1}$ \\ ${ }^{1}$ Department of Mechanical Engineering, Technical University of Denmark, \\ Nils Koppels Allé 404, DK-2800 Kgs. Lyngby, Denmark \\ 2 Department of Photonics Engineering, DTU Fotonik, Technical University of Denmark, \\ Building 343, DK-2800 Kgs. Lyngby, Denmark \\ *corresponding author, E-mail: raelch@mek.dtu.dk
}

\begin{abstract}
The preliminary study reported here investigates if unit-cell inclusion-symmetries may be broken in time-reversalinvariant topological insulator designs, while maintaining the desired global behaviour of pseudo-spin-dependent edge state based bi-directional, back-scattering robust, energy propagation. By allowing symmetries to be broken additional geometrical design freedom is attained, which may turn out to enable an improvement of various performance measures such as bandwidth and field confinement. The particular study considers a time-reversal-invariant acoustic topological insulator design, designed using a modified version of our recently proposed topology optimization based method for designing photonic and acoustic topological insulators. ${ }^{1}$ This method relies on a carefully constructed model system combined with the application of density based topology optimization to design two carefully interfaced crystal phases to maximize the flow of energy through the system. Through simple modifications of the method, we demonstrate that it is possible to design structures with different symmetry conditions from those that have previously been investigated using the method.
\end{abstract}

keywords: topological insulators, topology optimization, nano photonics, acoustics, meta materials, band gap structures

\section{INTRODUCTION}

It is well known that time-reversal-invariant topological insulators (TIs) support pseudo-spin-dependent edge states and that these states support spin-dependent directional energy propagation ${ }^{3,4}$ capable of suppressing back-scattering, as illustrated in Figure 1. It is also known that such TI-structures exhibit transmission robustness against certain classes of disorder. These properties potentially prove interesting for a range of applications where controlled propagation and low losses are essential. TIs can be created by carefully interfacing two crystal structures with tailored material distributions, each exhibiting C6v-symmetry. The design of such structures is however non-trivial since it requires the inversion of band symmetries around a band gap. Designing TI-structures may be done using bottom up approach relying on certain design rules and finely tuned intuition coupled with meticulous trial and error. ${ }^{5-8}$ However, given the vast parameter space for the material distributions constituting the two crystal phases, the use of intuition is unlikely to result in optimal TI-structures, hereby potentially leaving a large performance gap untapped. Therefore, the topology optimization ${ }^{9-11}$ based approach proposed in $^{1}$ for designing acoustic topological insulators (ATI) and later applied in ${ }^{2}$ to design a photonic topological insulator (PTI), offers a promising alternative to design and optimize the performance of novel TI-structures. The approach allows for tailoring TI-structures for different figures of merit such as the operational wavelength and/or the operational bandwidth; the crystal symmetry etc. In this work we consider the physics of acoustics to investigate if TI-structures breaking the C6v-symmetry, in terms of the material 
distribution in the unit-cells, may be designed using the proposed approach. Breaking such symmetries means a greater geometrical design freedom, which may potentially allow for an improvement of performance measures. This study solely serves as a proof of concept, investigating if symmetries may be broken, and does thus not target or investigate the improvement of any particular performance measure.

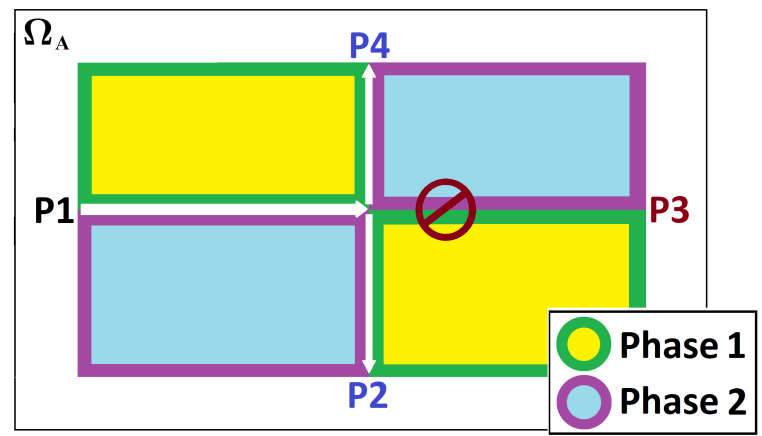

Figure 1: A conceptual sketch of the design problem used for designing a two crystal structure supporting pseudo-spin dependent edge-state propagation. The edge (bulk) of the two crystal phases constituting the TI-structure are colored magenta (blue) and green (yellow). The input/output ports are denoted P1-P4 with the white arrows indicating the power flow through the structure.

\section{METHOD}

The design problem treated in this work considers a model domain $\Omega_{\mathrm{A}} \in \mathbb{R}^{2}$, sketched in Figure 2a, with the physics of interest being modelled using a Helmholtz-type equation,

$$
\nabla \cdot\left(\mathrm{C}_{1} \frac{\omega_{0}-\mathrm{i} c \alpha}{\omega_{0}} \nabla \Psi\right)+\mathrm{C}_{2} \frac{\left(\omega_{0}-\mathrm{i} c \alpha\right)^{3}}{\omega_{0}} \Psi=-S_{\mathrm{e}}
$$

with $\Psi$ denoting the state field, $\omega_{0}=2 \pi \nu$ the free-space angular frequency with $\nu$ being the frequency, $\alpha$ denoting an attenuation parameter, $\mathrm{C}_{i}, \quad i \in\{1,2\}$ a set of material parameters and $S_{\mathrm{e}}$ a source. The two-dimensional model domain $\Omega_{\mathrm{A}} \in \mathbb{R}^{2}$ is truncated using a perfectly matched layer. ${ }^{12}$ Equation (1) may be used to model electromagnetic problems in $2 \mathrm{D}$ under the assumption of $\mathrm{TE}(\mathrm{TM})$ polarized light by taking $\left\{\mathrm{C}_{1}, \mathrm{C}_{2}\right\}=\left\{\frac{1}{\mu_{r}}, \epsilon_{r}\right\}$ $\left(\left\{\mathrm{C}_{1}, \mathrm{C}_{2}\right\}=\left\{\mu_{r}, \frac{1}{\epsilon_{r}}\right\}\right)$ and $\Psi=\operatorname{text} E_{z}\left(\Psi=\operatorname{text} B_{z}\right)$ with $\mu_{r}$ being the relative permeability, $\epsilon_{r}$ being the relative permittivity and $\operatorname{text} E_{z}\left(\operatorname{text} B_{z}\right)$ denoting the electric(magnetic) field. In this work we instead consider an acoustic problem, which may be modelled by taking $\left\{\mathrm{C}_{1}, \mathrm{C}_{2}\right\}=\left\{\frac{1}{\rho}, \frac{1}{\kappa}\right\}$ and $\Psi=p$ where $\rho$ denotes the material density, $\kappa$ the bulk modulus and $p$ the acoustic pressure.

The procedure proposed in ${ }^{1}$ is used to design the ATI-structure. In this procedure, a top-down approach is taken where the inverse design technique of topology optimization is used to determine the material distribution in each of two periodic hexagonal crystal phases (placed in $\Omega_{\mathrm{dR}, 1}$ and $\boldsymbol{\Omega}_{\mathrm{dR}, 2}$ ), designed to constitute a TI-structure by solving a transmission problem through the model domain sketched in Figure 2a and 2b. Taking as the objective of the design procedure, to achieve the global transmission behaviour illustrated in Figure 1, it is possible to design structures supporting pseudo-spin-dependent bi-directional edge-states, robust towards back-scattering. That is, the objective is taken as the maximization of transmission of the field emitted from the source $S_{\mathrm{e}}$ at port P1 through the structure under design, to ports P2 (blue in Figure 2b) and P4 (green in Figure 2b) while simultaneously minimizing the transmission to port P3 (red in Figure 2b) and requiring a band-gap to exist in the bulk of both crystal phases (grey in Figure 2b). 


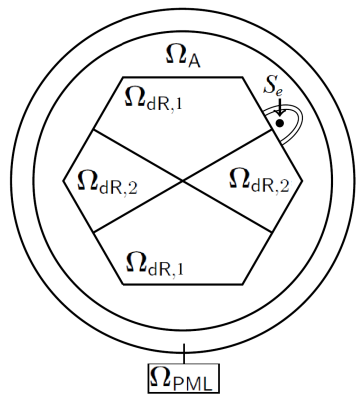

(a)

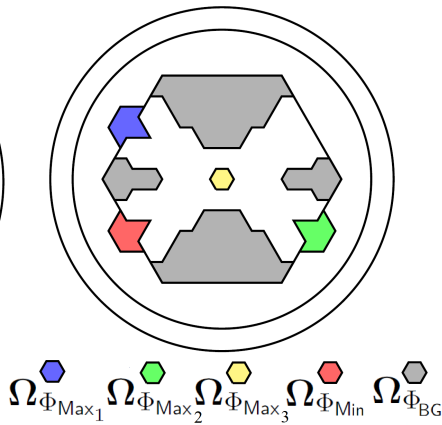

(b)

Figure 2: (a) The model domain $\boldsymbol{\Omega}_{\mathrm{A}} \in \mathbb{R}^{2}$ truncated using PML inside $\boldsymbol{\Omega}_{\mathrm{PML}}$, with two subdomains, $\boldsymbol{\Omega}_{\mathrm{dR}, 1}$ and $\boldsymbol{\Omega}_{\mathrm{dR}, 2}$ each containing a different crystal phase. $S_{\mathrm{e}}$ denotes a point source. (b) Sketch of the model domain with colors outlining different spatial regions used when formulating the design problem.

In difference to the work in $^{1}$ a number of symmetries enforced in that work in the hexagonal unit-cell are broken here. Instead of assuming C $3 \mathrm{v}$-symmetry in the design unit-cell, the simple translational symmetry illustrated in Figure 3 is imposed. That is, effectively a third of the hexagonal crystal cell is freely designable for both crystal phases and the design is then translated throughout the cell as illustrated in Figure 3c. This means, that instead of only designing one 36th of the total hexagonal unit cell as was done in, ${ }^{1}$ now one 3 rd of the unit-cell is designable.

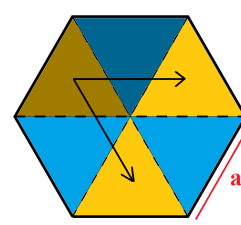

(a)

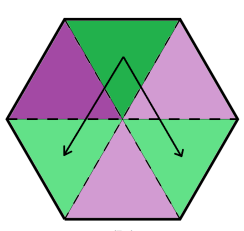

(b)

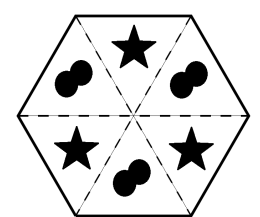

(c)

Figure 3: Illustration of the imposed translational symmetry in both the crystal phases (a) and (b). The dark colors yellow and blue (magenta and green) illustrates the designable regions in each phase, which is translated to the light colors as illustrated in (c).

For the example treated in the following the material parameters of the inclusions are taken to be those for aluminium, $\rho_{\text {aluminium }}=2643 \mathrm{~kg} \mathrm{~m} \mathrm{~m}^{-3}, \kappa_{\text {aluminium }}=6.87 \cdot 10^{10} \mathrm{~N} \mathrm{~m}^{-2}$ and the background medium is taken to be air, $\rho_{\text {air }}=1.204 \mathrm{~kg} \mathrm{~m}^{-3}, \kappa_{\text {air }}=1.42 \cdot 10^{5} \mathrm{~N} \mathrm{~m}^{-2}$. The targeted frequency is taken to be $\nu=20 \mathrm{kHz}, \mathrm{a}$ lattice constant of $a=0.01 \mathrm{~m}$ is used (see Figure 3a) and the source, $S_{\mathrm{e}}$ is taken to be an acoustic monopole.

The physics is modelled in COMSOL Multiphysics 5.3 where the design problem is also solved using the deterministic gradient-based optimization method, the globally convergent method of moving asymptotes (GCMMA). ${ }^{14}$

After the design process is completed, the band-structure and associated mode profiles for a supercell containing both crystal phases and their interface is computed, see Figure 7. This is done to validate that a pair of counter-rotating 
pseudo-spin edge states exists inside the bulk-bandgap region at the targeted frequency, supporting the successful design of a time-reversal invariant topological insulator.

\section{DISCUSSION}

An two phase structure is designed under the symmetry condition illustrated in Figure 3 using the proposed inverse design approach. The structure is designed starting from the material distribution shown in Figure 4a. Both crystal phases and the interface between them for the design are shown in Figure 4b. From this figure it is clearly seen that neither crystal phase exhibit C6v-symmetry.

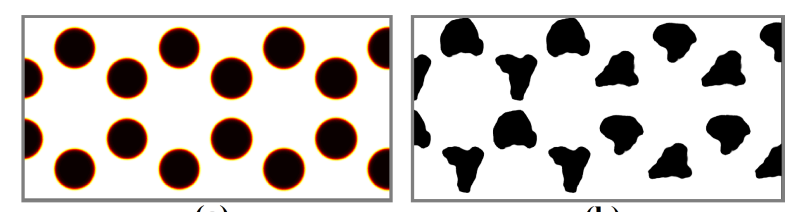

(a)

(b)

Figure 4: The (a) initial and (b) final distribution of aluminium (black) and air (white) for the PCs constituting the TI. The broken symmetries in each crystal phase in the final design are clearly observed.

Figure 5a/5b shows the acoustic pressure field in dB SPL for the initial/final material distribution in Figure 4a/4b when excited by a monopolar source placed at port P1. From these plots it is clear to see that the initial material configuration constitutes a band-gab crystal with a rapid decay of the pressure field emitted from the source, $S_{\mathrm{e}}$, at P1 as it extends into the crystal structure. For the final configuration in Figure $4 \mathrm{~b}$ the desired global behavior is clearly observed with the vast majority ( $>99 \%$ ) of the acoustic power coupled into the structure at P1 being transmitted to ports $\mathrm{P} 2$ and $\mathrm{P} 4$. That is, a two phase crystal structure exhibiting edge-state transport of acoustic energy at the targeted frequency has been designed.

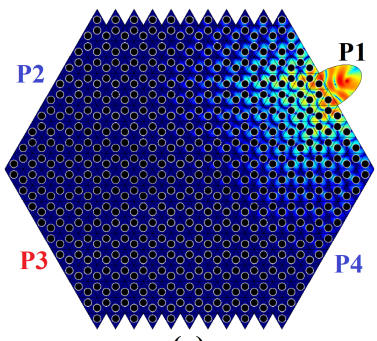

(a)

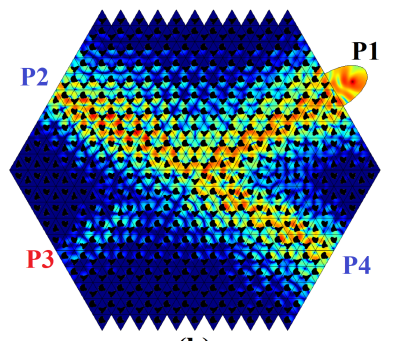

(b)

Figure 5: Max normalized acoustic pressure [30 dB SPL scale] (jet colormap) for the design model configuration considering (a) the initial and (b) the final distribution of aluminium (black).

To investigate if the edge-states carrying the acoustic energy are indeed back-scattering robust under certain types of defects, an interface is setup with and without a cavity defect, see Figure 6 . The structure is excited using a monopolar source, oscillating at $\nu=20 \mathrm{kHz}$, placed on the right side of the structure close to the interface 
between the two crystal phases. Figure 6 shows the structure (white) with the acoustic pressure field [on a $30 \mathrm{~dB}$ SPL scale] (jet colormap). While a significant change in the pressure field distribution near the cavity is observable it is not possible to visually observe a change in the transmitted pressure field on the left hand side of the structure.

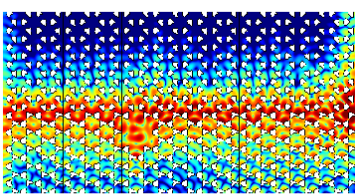

(a)

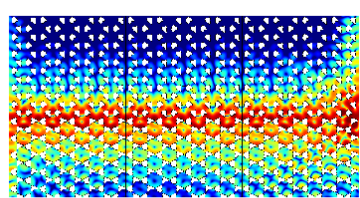

(b)

Figure 6: Acoustic pressure [30 dB SPL scale] (jet colormap) for field propagation from right to left at $\nu=20 \mathrm{kHz}$ along the TI-interface with (a) and without (b) a cavity.

Finally, it is investigated if a structure, which support a pair of psudo-spin edge-states inside a bulk-band gap region for both crystal phases, has been designed. To this end the band-structure, see Figure 7, for the super-cell shown to the right in Figure 7 has been computed. This band-structure clearly shows that two edge-states (bands) exists inside the bulk band-gap region (colored grey). The two bands have been colored according to their pseudo-spin clockwise (red) and counter-clockwise (blue).

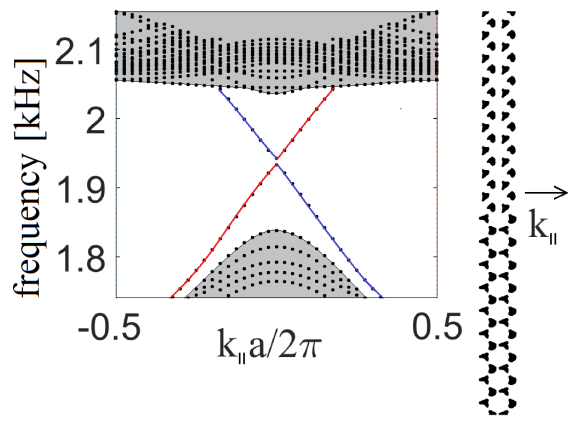

Figure 7: Band structure for the supercell (left) with the bulk band regions indicated in grey and the edge-states highlighted in red(blue) for positive(negative) pseudo-spin, respectively.

\section{CONCLUSION}

It is investigated whether a time-reversal invariant ATI design, breaking C6v-symmetry, can be designed using the inverse design approach proposed in. ${ }^{1}$ A structure is proposed, analysed and shown to exhibit a large bulk bandgap as well as psudo-spin dependent, back-scattering robust, bi-direction edge-state field propagation. This study thus suggests that it is possible to design TI-structures which do not conform to C6v-symmetry. 


\section{Acknowledgement}

The authors acknowledge support from NATEC (NAnophotonics for Terabit Communications) Centre (Grant No. 8692) and the Villum Foundation Young Investigator Programme.

\section{References}

[1] Christiansen, R. E., Wang, F., and Sigmund, O., "Topological Insulators by Topology Optimization”, Physical Review Letters 122, 234502, (2019).

[2] Christiansen, R. E., Wang, F., and Sigmund, O., and Stobbe, S., ’Designing photonic topological insulators with quantum-spin-Hall edge states using topology optimization" Nanophotonics, DOI: nanoph-2019-0057

[3] Lu, L., and Joannopoulos, J. D., and Soljačić, M., "Topological photonics" Nature photonics 8, 821-829, (2014).

[4] Khanikaev, A. B., and Shvets, G., "Two-dimensional topological photonics", Nature photonics 11, 763-773, (2017).

[5] Wu, L.-H., and Hu, X., "Scheme for Achieving a Topological Photonic Crystal by Using Dielectric Material", Physical Review Letters 114, 223901, (2015).

[6] Barik, S., Miyake, H., DeGottardi, W., Waks, E., and Hafezi, M., "Two-dimensionally confined edge states in photonic crystals", New Journal of Physics 18, 113013, (2016) .

[7] Ma, T., and Shvets, G., "All-Si valley-Hall photonic topological insulator", New Journal of Physics 18, 025012, (2016).

[8] He, C., Ni, X., Gu, H., Sun, X., Chen, Y., Lu, M., Liu, X., and Chen, Y., ”Acoustic topological insulator and robust one-way sound transport", Nature physics 12, 1124, (2016).

[9] Bendsøe, M. P., and Sigmund, O., Topology Optimization, Springer, (2003).

[10] Jensen, J. S., and Sigmund, O., "Topology optimization for nano-photonics", Laser \& Photonics Reviews 5, 308-321, (2011).

[11] Molesky, S., Lin, Z., Piggott, A. Y., Jin, W., Vucković, J., and Rodriguez, A. W., ’Inverse design in nanophotonics", Nature Photonics, 12, 659, (2018).

[12] Berenger, J.-P., "A Perfectly Matched Layer for the Absorption of Electromagnetic Waves", Journal of Computational Physics 114, 185-200, (1994).

[13] Lodahl, P., Mahmoodian, S., Stobbe, S., Rauschenbeutel, A., Schneeweiss, P., Volz, J., Pichler, H., and Zoller, P., "Chiral Quantum Optics", Nature 541, 473, (2017).

[14] Svanberg, K., "A Class of Globally Convergent Optimization Methods Based on Conservative Convex Separable Approximations", Siam Journal on Optimization, 12, 555, (2002). 